PROCEEDINGS OF THE

AMERICAN MATHEMATICAL SOCIETY

Volume 125, Number 12, December 1997, Pages 3569-3574

S 0002-9939(97)04100-2

\title{
DOMAINS OF FINITE TYPE AND HÖLDER CONTINUITY OF THE PERRON-BREMERMANN FUNCTION
}

\author{
DAN COMAN
}

(Communicated by Eric Bedford)

\begin{abstract}
Let $\Omega$ be a smoothly bounded domain in $\mathbb{C}^{n}$ such that $0 \in \partial \Omega$. We give a bound for the type of $\partial \Omega$ at 0 in terms of the Hölder exponent of its Perron-Bremermann function with simple boundary data. We then use this to show that a smoothly bounded domain in $\mathbb{C}^{2}$ is pseudoconvex of finite type if and only if its Perron-Bremermann function corresponding to Hölder continuous boundary data is Hölder continuous.
\end{abstract}

\section{INTRODUCTION}

Let $\Omega$ be a bounded domain in $\mathbb{C}^{n}$. A point $\zeta \in \partial \Omega$ is called a peak point for the class of plurisubharmonic functions if there exists a function $\rho \in P S H(\Omega) \cap C(\bar{\Omega})$ such that $\rho(\zeta)=0$ and $\rho(z)<0$ for all $z \in \bar{\Omega}, z \neq \zeta$. We now let $f \in C(\partial \Omega)$ and we denote by $U(\Omega, f)$ the set of plurisubharmonic functions $v$ on $\Omega$ satisfying $\lim \sup _{z \rightarrow \zeta, z \in \Omega} v(z) \leq f(\zeta)$, for all $\zeta \in \partial \Omega$. The Perron-Bremermann function $\Psi_{\Omega, f}$ for $\Omega$ and $f$ is defined by $\Psi_{\Omega, f}(z)=\sup \{v(z): v \in U(\Omega, f)\}, z \in \Omega$ (see for example [9, p. 89]). We can extend $\Psi_{\Omega, f}$ to $\bar{\Omega}$ by setting $\Psi_{\Omega, f}=f$ on $\partial \Omega$. So, in what follows, whenever $\Psi_{\Omega, f}$ is referred to as a function defined on $\bar{\Omega}$ we assume that it is extended to $\partial \Omega$ in this way. If $\Omega$ is regular for the Dirichlet problem for the Laplace operator it is easy to see that $\Psi_{\Omega, f} \in U(\Omega, f)$. A classical theorem of Walsh [11] states that if $\lim _{z \rightarrow \zeta, z \in \Omega} \Psi_{\Omega, f}(z)=f(\zeta)$ for all $\zeta \in \partial \Omega$ then $\Psi_{\Omega, f}$ is actually continuous on $\bar{\Omega}$. This fact implies the following well known theorem (see also Theorem 2.3 in [10]).

Theorem. Let $\Omega$ be a bounded domain in $\mathbb{C}^{n}$, regular for the Dirichlet problem for the Laplace operator. The following are equivalent:

(i) every $\zeta \in \partial \Omega$ is a peak point for the class of plurisubharmonic functions;

(ii) if $f \in C(\partial \Omega)$ then $\Psi_{\Omega, f} \in C(\bar{\Omega})$.

In [2] it is shown (Theorem 6.2) that if $\Omega$ is a strongly pseudoconvex domain in $\mathbb{C}^{n}$ and $f$ is a Hölder continuous function on $\partial \Omega$ then the function $\Psi_{\Omega, f}$ is Hölder continuous on $\bar{\Omega}$. Moreover, estimates for the modulus of continuity of $\Psi_{\Omega, f}$ in terms of the modulus of continuity of $f$ are obtained, via different methods, in [7] and [1]. In this note we prove that a smoothly bounded domain $\Omega$ in $\mathbb{C}^{2}$ is pseudoconvex of finite type if and only if the function $\Psi_{\Omega, f}$ is Hölder continuous on $\bar{\Omega}$ whenever $f$ is a Hölder continuous function on $\partial \Omega$.

Received by the editors July 1, 1996 .

1991 Mathematics Subject Classification. Primary 32F25, 32F05; Secondary 32F15.

(C)1997 American Mathematical Society 
For $S \subset \mathbb{C}^{n}$ and $\alpha$ so that $0<\alpha \leq 1$ we denote by $\operatorname{Lip}^{\alpha}(S)$ the set of functions on $S$ which are Hölder continuous with exponent $\alpha$. If $f$ is a real valued function defined on $S$ we set $H(f)=\sup \left\{\alpha \in(0,1]: f \in \operatorname{Lip}^{\alpha}(S)\right\}$. Note that if this set is empty then, by definition, $H(f)=0$. We also set, for a fixed $x_{0} \in S$,

$$
h\left(f, x_{0}\right)=\max \left\{\liminf _{x \rightarrow x_{0}, x \in S} \frac{\log \left|f(x)-f\left(x_{0}\right)\right|}{\log \left\|x-x_{0}\right\|}, 0\right\} .
$$

It is then obvious that if $f$ satisfies a Hölder condition at $x_{0}$ with exponent $\alpha>0$ (i.e. $\left|f(x)-f\left(x_{0}\right)\right| \leq c\left\|x-x_{0}\right\|^{\alpha}$ for $x$ in some neighborhood of $\left.x_{0}\right)$ then $\alpha \leq$ $h\left(f, x_{0}\right)$. In particular $H(f) \leq h\left(f, x_{0}\right)$. Conversely, if $h\left(f, x_{0}\right)>0$ then for any $\epsilon$, $0<\epsilon<h\left(f, x_{0}\right), f$ satisfies a Hölder condition at $x_{0}$ with exponent $h\left(f, x_{0}\right)-\epsilon$.

Let us briefly recall one of the definitions of the notion of finite type. Let $\Omega$ be a bounded domain in $\mathbb{C}^{n}$ with $C^{\infty}$ smooth boundary and assume $0 \in \partial \Omega$. If $X$ is a germ of a one-dimensional analytic variety at 0 we set

$$
\tau(X)=\sup \left\{s>0: \limsup _{z \rightarrow 0, z \in X \backslash\{0\}} \frac{d(z, \partial \Omega)}{\|z\|^{s}}<\infty\right\}
$$

( $d=$ euclidean distance). If $r$ is a defining function for $\partial \Omega$ near $0, X$ is irreducible and $z=\beta(t), t \in \mathbb{C}$, is a parametrization for $X$ such that $\beta(0)=0$, then it is easy to see that $\tau(X)=v(r \circ \beta) / v(\beta)$, where $v(r \circ \beta)$ and $v(\beta)$ are the vanishing orders of $r \circ \beta$ and $\beta$ at 0 . We say that $\partial \Omega$ is of type less than $2 k$ at 0 if $\tau(X) \leq 2 k$ for every germ $X$ of a one-dimensional analytic variety at 0 (for more information see [3]). The domain $\Omega$ is said to be of finite type if $\partial \Omega$ is of finite type at all of its points.

\section{MAIN RESUlts}

For $z \in \mathbb{C}^{n}$ we write $z=\left(z^{\prime}, z_{n}\right) \in \mathbb{C}^{n-1} \times \mathbb{C}, z_{n}=x_{n}+i y_{n}$. Let $\Omega$ be a bounded domain in $\mathbb{C}^{n}$ with $C^{\infty}$ smooth boundary and let $\zeta \in \partial \Omega$. Without loss of generality we may assume that $\zeta=0$ and that the boundary of $\Omega$ near 0 is given by $y_{n}=\lambda\left(z^{\prime}, x_{n}\right)$, where $\lambda$ is a smooth function satisfying $\lambda(0,0)=0, d \lambda(0,0)=0$.

We define $f_{1}(z)=-\left\|\left(z^{\prime}, x_{n}\right)\right\|=-\left(\left\|z^{\prime}\right\|^{2}+x_{n}^{2}\right)^{1 / 2}, z \in \mathbb{C}^{n}$, and we let $\psi_{1}(z)=$ $\Psi_{\Omega, f_{1}}(z), z \in \bar{\Omega}$, be the Perron-Bremermann function for $\Omega$ and $f_{1}$. Note that $H\left(f_{1}\right)=1$.

Theorem 1. Let $\Omega$ and $\psi_{1}$ be as above and let $X$ be a germ of a one-dimensional analytic variety at $0 \in \partial \Omega$. Then

$$
\tau(X) \leq 1 / h\left(\psi_{1}, 0\right) .
$$

Theorem 2. Let $\Omega$ be a bounded domain in $\mathbb{C}^{2}$ with $C^{\infty}$ boundary. The following are equivalent:

(i) $\Omega$ is pseudoconvex of finite type;

(ii) for any $f \in \operatorname{Lip}^{\alpha}(\partial \Omega), 0<\alpha \leq 1$, we have $\Psi_{\Omega, f} \in \operatorname{Lip}^{\gamma}(\bar{\Omega})$, for some $\gamma=\gamma(f, \alpha), 0<\gamma \leq \alpha$.

Moreover, if (i) holds and the type of $\Omega$ is $2 k$ then $\gamma$ can be chosen as $\gamma=\gamma(\alpha)=$ $\alpha /(2 k)$. In addition, if $\zeta \in \partial \Omega(w \log \zeta=0)$ is a point of the highest type $2 k$ and $\psi_{1}$ is as in Theorem 1 , then $H\left(\psi_{1}\right)=1 /(2 k)$. 


\section{Proofs}

We need the following lemma ([5, Proposition 3.1]):

Lemma. Let $\Omega$ be a smoothly bounded domain in $\mathbb{C}^{n}$ with $0 \in \partial \Omega$ and let $X$ be a germ of a one-dimensional analytic variety at 0 . Assume that there exists a negative plurisubharmonic function $\phi$ on $\Omega$ such that for some $r>0$ and for $z \in \Omega$, with $\|z\|<r$, $\phi$ satisfies $-c_{1}\|z\|^{\gamma} \leq \phi(z) \leq-c_{2}\|z\|^{N}$, where $c_{1}, c_{2}>0,0<\gamma<N$. Then $\tau(X) \leq N / \gamma$.

Proof of Theorem 1. We may assume that $h\left(\psi_{1}, 0\right)>0$. We fix $\epsilon$ so that $0<$ $\epsilon<h\left(\psi_{1}, 0\right)$, and set $\gamma=\gamma(\epsilon)=h\left(\psi_{1}, 0\right)-\epsilon$. Note that $\psi_{1}$ is negative and plurisubharmonic in $\Omega$ and by the definition of $h\left(\psi_{1}, 0\right)$ there is a number $r>0$ such that $\psi_{1}(z) \geq-\|z\|^{\gamma}$ for $z \in \Omega$ with $\|z\|<r$.

It is enough to prove that for every $\alpha<1$ there is a ball $U_{\alpha}$ centered at 0 such that

$$
\psi_{1}(z) \leq-c_{\alpha}\|z\|^{1 / \alpha}
$$

for $z \in \Omega \cap U_{\alpha}$. Indeed, then it would follow from the above lemma that $\tau(X) \leq$ $1 /(\alpha \gamma(\epsilon))$, and hence $\tau(X) \leq 1 / h\left(\psi_{1}, 0\right)$ by letting $\epsilon \rightarrow 0$ and $\alpha \rightarrow 1$.

To this end we introduce the functions $f_{\alpha}(z)=-\left\|\left(z^{\prime}, x_{n}\right)\right\|^{\alpha}=-\left(\left\|z^{\prime}\right\|^{2}+x_{n}^{2}\right)^{\alpha / 2}$, $z \in \mathbb{C}^{n}$, and $\psi_{\alpha}(z)=\Psi_{\Omega, f_{\alpha}}(z), z \in \bar{\Omega}$. Note that $f_{\alpha} \in \operatorname{Lip}^{\alpha}\left(\mathbb{C}^{n}\right), H\left(f_{\alpha}\right)=\alpha$ and $\psi_{\alpha}$ is negative and plurisubharmonic in $\Omega$. As $\alpha<1$ the function $v=-\left(-\psi_{1}\right)^{\alpha}$ is plurisubharmonic in $\Omega$. Also since $\lim \sup _{z \rightarrow \zeta} \psi_{1}(z) \leq f_{1}(\zeta), \zeta \in \partial \Omega$, we have $\lim \sup _{z \rightarrow \zeta} v(z) \leq-\left(-f_{1}(\zeta)\right)^{\alpha}=f_{\alpha}(\zeta)$, so

$$
v(z)=-\left(-\psi_{1}(z)\right)^{\alpha} \leq \psi_{\alpha}(z), z \in \Omega .
$$

Thus (3.1) follows from (3.2) if we show that

$$
\psi_{\alpha}(z) \leq-c_{\alpha}\|z\|,
$$

for some constant $c_{\alpha}>0$ and for $z \in \Omega \cap U_{\alpha}, U_{\alpha}$ being a suitable ball centered at 0.

We fix $\delta>0$ small, to be specified later in terms of $\alpha$ alone, and write $\Omega=$ $\Omega_{1} \cup \Omega_{2}$ near 0 , where $\Omega_{1}=\left\{z \in \Omega:\|z\|<r(\delta),\left|y_{n}\right| \geq \delta\left(\left\|z^{\prime}\right\|^{2}+x_{n}^{2}\right)^{1 / 2}\right\}$, $\Omega_{2}=\left\{z \in \Omega:\|z\|<r(\delta),\left|y_{n}\right| \leq \delta\left(\left\|z^{\prime}\right\|^{2}+x_{n}^{2}\right)^{1 / 2}\right\}$ and $r(\delta)$ is sufficiently small. By Hopf's lemma ([6, p. 57]) there is a constant $c>0$ depending on $\Omega$ and $\psi_{\alpha}$ such that $\psi_{\alpha}(z) \leq-c d(z, \partial \Omega)$. But for $z \in \Omega_{1}$ we have $d(z, \partial \Omega) \geq c(\delta)\|z\|$ provided $r(\delta)$ is small enough; hence (3.3) holds if $z \in \Omega_{1}$.

Now let $h$ be the harmonic function in $\Omega$ with boundary value $f_{\alpha}$. Since $f_{\alpha} \in \operatorname{Lip}^{\alpha}$ we have as a consequence of the results in [8] that $h \in \operatorname{Lip}^{\alpha}(\bar{\Omega})$ (note that this is not necessarily true when $\alpha=1$ ). Let $k(\alpha)>0$ be such that $|h(z)-h(w)| \leq k(\alpha)\|z-w\|^{\alpha}, z, w \in \bar{\Omega}$. For $z=\left(z^{\prime}, x_{n}+i y_{n}\right) \in \Omega$ near 0 we define $\widetilde{z}=\left(z^{\prime}, x_{n}+i \widetilde{y}_{n}\right) \in \partial \Omega$. So $\widetilde{y}_{n}=\lambda\left(z^{\prime}, x_{n}\right)$ and hence $\left|\widetilde{y}_{n}\right| \leq l\left(\left\|z^{\prime}\right\|^{2}+x_{n}^{2}\right)$ for some constant $l>0$ depending on $\lambda$. For $z \in \Omega_{2}$ we have:

$$
\begin{aligned}
\psi_{\alpha}(z) & \leq h(z) \leq f_{\alpha}(\widetilde{z})+k(\alpha)\left|y_{n}-\widetilde{y}_{n}\right|^{\alpha} \\
& \leq-\left(\left\|z^{\prime}\right\|^{2}+x_{n}^{2}\right)^{\alpha / 2}+k(\alpha)\left|y_{n}\right|^{\alpha}+k(\alpha)\left|\widetilde{y}_{n}\right|^{\alpha} \\
& \leq-\left(\left\|z^{\prime}\right\|^{2}+x_{n}^{2}\right)^{\alpha / 2}+k(\alpha) \delta^{\alpha}\left(\left\|z^{\prime}\right\|^{2}+x_{n}^{2}\right)^{\alpha / 2}+k(\alpha) l^{\alpha}\left(\left\|z^{\prime}\right\|^{2}+x_{n}^{2}\right)^{\alpha} .
\end{aligned}
$$


We now choose $\delta=\delta(\alpha)$ small enough so that $k(\alpha) \delta^{\alpha} \leq 1 / 4$ and also choose $r(\delta)$ small enough so that $k(\alpha) l^{\alpha}\left(\left\|z^{\prime}\right\|^{2}+x_{n}^{2}\right)^{\alpha / 2} \leq 1 / 4$. Thus by the above

$$
\psi_{\alpha}(z) \leq-\frac{1}{2}\left(\left\|z^{\prime}\right\|^{2}+x_{n}^{2}\right)^{\alpha / 2}
$$

for $z \in \Omega_{2}$. Also, for $z \in \Omega_{2}$ we have

$$
\|z\|^{2} \leq\left(1+\delta^{2}\right)\left(\left\|z^{\prime}\right\|^{2}+x_{n}^{2}\right) \leq 2\left(\left\|z^{\prime}\right\|^{2}+x_{n}^{2}\right) .
$$

Hence $\psi_{\alpha}(z) \leq-\frac{1}{4}\|z\|^{\alpha} \leq-\frac{1}{4}\|z\|$ for $z \in \Omega_{2}$, and (3.3) is proved.

Proof of Theorem 2. The implication (ii) $\Rightarrow$ (i) follows immediately from Theorem 1. Indeed, we pick a point on $\partial \Omega$ which without loss of generality may be assumed to be 0 . With the notations in Theorem 1 we have $f_{1} \in \operatorname{Lip}^{1}$ so $\psi_{1} \in \operatorname{Lip}^{\gamma}(\bar{\Omega})$, for some $\gamma \in(0,1)$, and hence $\tau(X) \leq 1 / \gamma$ for any germ $X$ of a one-dimensional analytic variety at 0 . Also, for any $\zeta \in \partial \Omega$, we let $f(z)=-\|z-\zeta\|$ and note that by (ii) $\Psi_{\Omega, f} \in P S H(\Omega) \cap C(\bar{\Omega})$ peaks at $\zeta$, so $\Omega$ is pseudoconvex.

To prove that (i) $\Rightarrow$ (ii) we follow the method of Bedford and Taylor ([2, Theorem $6.2]$ ), and use a theorem of Fornaess and Sibony ([5, Theorem A $]$ ) on the existence of plurisubharmonic peak functions. Precisely, if $\Omega$ is a bounded pseudoconvex domain in $\mathbb{C}^{2}$ with smooth boundary and of finite type $2 k$ then there exist a neighborhood $U$ of $\bar{\Omega}$ and a constant $C$ such that for each $\zeta \in \partial \Omega$ there is a plurisubharmonic function $\phi_{\zeta}(z)$ on $U$ satisfying $\left|\phi_{\zeta}(z)-\phi_{\zeta}(w)\right| \leq C\|z-w\|$ for $z, w \in U, \phi_{\zeta}(z) \leq-\|z-\zeta\|^{2 k}$ for $z \in \Omega$, and $\phi_{\zeta}(\zeta)=0$.

Now let $f \in \operatorname{Lip}^{\alpha}(\partial \Omega), 0<\alpha \leq 1$, and let $c_{f}$ be the smallest constant for which $|f(\zeta)-f(\xi)| \leq c_{f}\|\zeta-\xi\|^{\alpha}, \zeta, \xi \in \partial \Omega$. We fix $\zeta \in \partial \Omega$ and consider the function $v_{\zeta}(z)=-c_{f}\left(-\phi_{\zeta}(z)\right)^{\alpha /(2 k)}+f(\zeta)$. Since $\phi_{\zeta}$ is negative and plurisubharmonic in $\Omega$ and $\alpha /(2 k)<1$ the function $v_{\zeta}$ is plurisubharmonic in $\Omega$ and continuous on $\bar{\Omega}$. For $\xi \in \partial \Omega$ we have

$$
v_{\zeta}(\xi) \leq-c_{f}\|\xi-\zeta\|^{\alpha}+f(\zeta) \leq f(\xi),
$$

so $v_{\zeta}(z) \leq \Psi_{\Omega, f}(z)$, for $z \in \bar{\Omega}$. Note that $v_{\zeta}$ satisfies a Hölder condition of the form $\left|v_{\zeta}(z)-v_{\zeta}(w)\right| \leq C^{\prime} c_{f}\|z-w\|^{\alpha /(2 k)}, z, w \in \bar{\Omega}$, where $C^{\prime}=C^{\alpha /(2 k)}$.

We next define $u(z)=\sup \left\{v_{\zeta}(z): \quad \zeta \in \partial \Omega\right\}, z \in \bar{\Omega}$. Then $u$ satisfies the same Hölder condition as $v_{\zeta}$ so, in particular, $u$ is continuous on $\bar{\Omega}$ and hence plurisubharmonic in $\Omega$. Also $u(z) \leq \Psi_{\Omega, f}(z)$ for $z \in \bar{\Omega}$. As $v_{\zeta}(\xi) \leq f(\xi), \xi \in \partial \Omega$, and $v_{\xi}(\xi)=f(\xi)$ we see that $u=\Psi_{\Omega, f}=f$ on $\partial \Omega$. So if $z \in \bar{\Omega}$ and $\zeta \in \partial \Omega$ we have $-C^{\prime} c_{f}\|z-\zeta\|^{\alpha /(2 k)} \leq u(z)-u(\zeta) \leq \Psi_{\Omega, f}(z)-f(\zeta)$. Replacing $f$ by $-f$ and proceeding in the same way we construct a function $\widetilde{u}$ which satisfies the same Hölder condition as $u$ in $\bar{\Omega}$, is plurisubharmonic in $\Omega$ and $\widetilde{u}=-f$ on $\partial \Omega$. By the maximum principle $\Psi_{\Omega, f}+\widetilde{u} \leq 0$ on $\bar{\Omega}$ so for $z \in \bar{\Omega}$ and $\zeta \in \partial \Omega$ we get $\Psi_{\Omega, f}(z)-f(\zeta) \leq-\widetilde{u}(z)+\widetilde{u}(\zeta) \leq C^{\prime} c_{f}\|z-\zeta\|^{\alpha /(2 k)}$. We conclude that

$$
\left|\Psi_{\Omega, f}(z)-\Psi_{\Omega, f}(\zeta)\right| \leq C^{\prime} c_{f}\|z-\zeta\|^{\alpha /(2 k)},
$$

for $z \in \bar{\Omega}$ and $\zeta \in \partial \Omega$.

We now show that the above inequality holds for all $z, w \in \bar{\Omega}$. For $y \in \mathbb{C}^{n}$ we set $\Omega_{y}=\Omega \cap(-y+\Omega)$ and consider the function

$$
F_{y}(z)= \begin{cases}\max \left\{\Psi_{\Omega, f}(z), \Psi_{\Omega, f}(z+y)-C^{\prime} c_{f}\|y\|^{\alpha /(2 k)}\right\}, & \text { if } z \in \Omega_{y}, \\ \Psi_{\Omega, f}(z), & \text { if } z \in \Omega \backslash \Omega_{y} .\end{cases}
$$


By (3.4) we have $\lim \sup _{z \rightarrow \zeta, z \in \Omega} F_{y}(z) \leq f(\zeta)$ for all $\zeta \in \partial \Omega$. Also if $z \in \partial \Omega_{y} \cap \Omega$ then $z+y \in \partial \Omega$ so by (3.4) $\Psi_{\Omega, f}(z) \geq \Psi_{\Omega, f}(z+y)-C^{\prime} c_{f}\|y\|^{\alpha /(2 k)}$ and $F_{y}$ is plurisubharmonic. Hence $F_{y}(z) \leq \Psi_{\Omega, f}(z)$ for all $z \in \Omega$. If $w \in \Omega$ then $z \in \Omega_{w-z}$ so

$$
\Psi_{\Omega, f}(w)-C^{\prime} c_{f}\|w-z\|^{\alpha /(2 k)} \leq F_{w-z}(z) \leq \Psi_{\Omega, f}(z)
$$

and (3.4) holds for all $z, w \in \bar{\Omega}$.

Finally, we assume that $\zeta=0 \in \partial \Omega$ is a point of type $2 k$ and we let $\psi_{1}$ be the function in Theorem 1 . Then $1 /(2 k) \leq H\left(\psi_{1}\right) \leq h\left(\psi_{1}, 0\right) \leq 1 /(2 k)$, the last inequality following from Theorem 1 . Hence $H\left(\psi_{1}\right)=1 /(2 k)$ and the proof is complete.

Remark. If $f \in C(\partial \Omega)$ then it is easy to see that $\left\|\Psi_{\Omega, f}\right\|_{\infty} \leq\|f\|_{\infty}$ (indeed, $-2\|f\|_{\infty} \leq \Psi_{\Omega, f-\|f\|_{\infty}}=\Psi_{\Omega, f}-\|f\|_{\infty} \leq 0$, by the maximum principle). So if $\Omega$ is a bounded pseudoconvex domain in $\mathbb{C}^{2}$ of finite type $2 k$ it follows from the proof of the above theorem that there exists a constant $C$ depending only on $\Omega$ such that, for $f \in \operatorname{Lip}^{\alpha}(\partial \Omega), 0<\alpha \leq 1$, we have $\left\|\Psi_{\Omega, f}\right\|_{\frac{\alpha}{2 k}} \leq C\|f\|_{\alpha}$. Here $\|\cdot\|_{\alpha}$ denotes the standard norm on $\operatorname{Lip}^{\alpha}(S),\|f\|_{\alpha}=\|f\|_{\infty}+\sup \left\{|f(x)-f(y)| /\|x-y\|^{\alpha}: x \neq y \in S\right\}$.

We finally consider the case when $\Omega$ is a bounded pseudoconvex domain in $\mathbb{C}^{n}$ with real analytic boundary. It follows from Theorems 2 and 3 in [4] that there are constants $C, \gamma$ and $N$ depending only on $\Omega$ such that $C>0,0<\gamma<1<N$, and such that for every $\zeta \in \partial \Omega$ there is a function $\phi_{\zeta} \in P S H(\Omega) \cap C(\bar{\Omega})$ satisfying $\left|\phi_{\zeta}(z)-\phi_{\zeta}(w)\right| \leq C\|z-w\|^{\gamma}$ for $z, w \in \bar{\Omega}, \phi_{\zeta}(z) \leq-\|z-\zeta\|^{N}$ for $z \in \Omega$ and $\phi_{\zeta}(\zeta)=0$. Using these plurisubharmonic peak functions one can proceed as in the proof of Theorem 2 to obtain the following

Theorem 3. Let $\Omega$ be a bounded pseudoconvex domain in $\mathbb{C}^{n}$ with real analytic boundary. Then there exists a constant $m>1$ depending only on $\Omega(m=N / \gamma$, where $\gamma$ and $N$ are as above) such that if $f \in \operatorname{Lip}^{\alpha}(\partial \Omega), 0<\alpha \leq 1$, then $\Psi_{\Omega, f} \in$ $\operatorname{Lip}^{\alpha / m}(\bar{\Omega})$.

\section{REFERENCES}

[1] E. Bedford, Levi flat hypersurfaces in $\mathbb{C}^{2}$ with prescribed boundary: stability, Annali della Scuola Normale Superiore di Pisa, 9(1982), 529-570. MR 85d:32029

[2] E. Bedford and B. A. Taylor, The Dirichlet problem for a complex Monge-Ampére equation, Inventiones Mathematicae, 37(1976), 1-44. MR 56:3351

[3] J. D'Angelo, Real hypersurfaces, orders of contact and applications, Annals of Mathematics, 115(1982), 615-637. MR 84a:32027

[4] K. Diederich and J. E. Fornaess, Proper holomorphic maps onto pseudoconvex domains with real analytic boundary, Annals of Mathematics, 110(1979), 575-592. MR 81h:32027

[5] J. E. Fornaess and N. Sibony, Construction of psh functions on weakly pseudoconvex domains, Duke Mathematical Journal, 58(1989), 633-655. MR 90m:32034

[6] J. E. Fornaess and B. Stensones, Lectures on Counterexamples in Several Complex Variables, Princeton University Press, Princeton, NJ, 1987. MR 88f:32001

[7] B. Gaveau, Méthodes de contrôle optimal en analyse complexe: résolution d'équations de Monge-Ampère, Journal of Functional Analysis, 25(1977), 391-411. MR 56:15987

[8] D. Gilbarg and L. Hörmander, Intermediate Schauder estimates, Archive for Rational Mechanics and Analysis, 74(1980), 297-318. MR 82a:35038

[9] M. Klimek, Pluripotential Theory, Clarendon Press, Oxford New York Tokyo, 1991. MR 93h:32021 
[10] N. Sibony, Some aspects of weakly pseudoconvex domains, Proceedings of Symposia in Pure Mathematics, vol 52(1991), Part 1, 199-231. MR 92g:32034

[11] J. B. Walsh, Continuity of envelopes of plurisubharmonic functions, Journal of Mathematics and Mechanics, 18(1968), 143-148. MR 37:3049

Department of Mathematics, University of Michigan, Ann Arbor, Michigan 481091109

E-mail address: dan.coman@math.lsa.umich.edu

Current address: Department of Mathematics, University of Notre Dame, Notre Dame, Indiana 46556 